« Monnaie et Crise Bancaire dans une Petite Economie Ouverte"

\author{
$\underline{\text { Auteurs }}$
}

Jin Cheng

Document de Travail n 2010 - 02

Janvier 2010

Faculté des sciences économiques et de gestion

Pôle européen de gestion et d'économie (PEGE) 61 avenue de la Forêt Noire F-67085 Strasbourg Cedex

Secétariat du BETA Géraldine Manderscheidt Tél. : (33) 0368852069 Fax : (33) 0368852070 g.manderscheidt@unistra.fr http://cournot2.u-strasbg.fr/beta 


\title{
Monnaie et Crise Bancaire dans une Petite Economie Ouverte
}

\author{
Jin CHENG*
}

Résumé : Cet article développe un modèle intégrant le rôle de la monnaie et de l'IDE afin d'examiner la fragilité du système bancaire d'une petite économie ouverte dans un contexte de crise financière internationale. Le modèle met l'accent sur les décalages de maturité et de devise au niveau du bilan des banques, et il illustre les impacts de la contraction du crédit sur le marché financier international, via les banques commerciales, sur la monnaie et la production du pays. Cet article analyse également les options de politique économique de la petite économie permettant d'atténuer les effets négatifs de la fuite des capitaux étrangers, ainsi que d'éviter l'illiquidité internationale de son système bancaire.

Mots clefs : Ruée bancaire; taux d'intérêt; taux de change; capital; dette étrangère; politique macroéconomique.

JEL classification: G2; E43; F31; E22; F34; F4.

\section{Money and banking crisis in a small open economy}

Abstract: This paper develops an analytical framework integrating the money and the FDI for evaluating the problem of the banking system of a small open economy during an international financial crisis. The framework draws attention to the vulnerabilities created by the maturity and currency mismatches of bank's balance sheet, and it makes transparent how the credit crunch in foreign countries can affect the value of country's money and its economic activity through commercial banks. The paper discusses as well the potential of macroeconomic policies and official intervention to alleviate the negative influence of capital outflows and thereupon to avoid the illiquidity of the country's bank system.

Keywords: Bank run, interest rates, exchange rate, capital, foreign debt, macroeconomic policy.

* BETA, l'Unitersité de Strasbourg, 67, avenue de la Forêt Noire, 67000 Strasbourg, France. Emai : cheng@unistra.fr. 


\section{Introduction}

La crise financière actuelle a mis en évidence les problèmes que certaines petites économies ont accumulés en libéralisant rapidement leur secteur financier. Malgré leurs grandes différences, la situation de crise à laquelle l'Islande et certains pays d'Europe centrale et orientale (PECO) sont confrontés est comparable à celle de leurs homologues asiatiques en 1997. ${ }^{1}$ En effet, la croissance rapide de l'activité de ces pays est nourrie surtout par des afflux massifs du crédit international. En plus, une fraction très importante du financement est basée sur le recours à l'emprunt étranger à court terme avec une très grande majorité d'obligations libellées en devise forte. Enfin, tous ces pays (sauf l'Islande) ont un régime de change plus ou moins rigide. A l'instar des pays asiatiques à l'époque de la crise, le système financier de l'Islande et des PECO a connu une dépendance accrue vis-à-vis des créances internationales et une vulnérabilité aggravée face aux chocs externes.

Une réduction 'inopinée' des afflux des capitaux étrangers dans une petite économie lourdement endettée à court terme entraîne une contraction violente du crédit et l'effondrement brutal du système financier du pays, qui mènera immédiatement à une dépréciation forte de la monnaie nationale et à une récession profonde du secteur réel. Ainsi, ce 'sudden stop' des afflux de capitaux étrangers peut déclencher un cercle vicieux entre le secteur réel affaibli, le système financier détérioré et la monnaie nationale dépréciée. Sans une politique économique adaptée et des aides internationales, les pays peuvent entrer dans une récession profonde et prolongée.

A la différence de la crise asiatique de 1997, dans les PECO, les grandes banques et les

\footnotetext{
${ }^{1}$ Voir Buiter et Sibert (2009) et Danielsson (2009) pour une description détaillée de la crise Islandaise. Voir Sirtaine et Skamnelos (2007) et Von Hagen et Siedschlag (2008) pour celle de PECO.
} 
avoirs bancaires sont principalement détenus par les banques d'Europe occidentale à travers l'investissement direct étranger (l'IDE). Pourtant, la solidité de leurs systèmes financiers ne s'est pas améliorée de façon considérable par rapport à ceux d'Asie en 1997. Un choc défavorable (la crise des subprimes par exemple) sur l'une ou plusieurs banques occidentales pourrait se traduire directement par des retraits de fonds dans les petits pays. Selon le FMI, en moyenne, le système bancaire des PECO est cinq fois plus exposé que les banques américaines ou japonaises, et leur effet de levier est supérieur de 50\%.

Dans la crise récente, l'Islande a connu effectivement une crise majeure, avec son système bancaire dévasté et une économie en récession sévère. Quant aux PECO, la BERD pronostique un taux de croissance négative en 2009 et en 2010. Pour l'instant, L'Islande, la Hongrie et la Lettonie ont demandé l'aide d'urgence au FMI.

Beaucoup d'économistes (Chari et Jagganathan (1988), Calomiris et Kahn (1991), Diamond et Dybvig (1983) et Hellwing (1991)) ont porté leur attention sur le rôle de la vulnérabilité de la structure du capital bancaire dans le déclenchement de la crise financière.

Suite à la crise asiatique en 1997, certains économistes (voir par exemple: Hardy et Pazarbasioglu (1998), Kaminsky et Reinhart, (1999), Kaminsky (1998), et Chang et Velasco (2001)) considèrent que la crise asiatique résulte de la fragilité financière et de l'illiquidité internationale et suggèrent que les crises de change sont le sous-produit d'une ruée bancaire. Cette dernière est modélisée par Diamond et Dybvig (1983) comme étant une autoréalisation de la perte de confiance forçant les intermédiaires financiers à liquider leurs investissements de manière prématurée. Le modèle de Chang et Vélasco (2001) fournit une version d'économie ouverte du modèle de Diamond et Dybving (1983). Il nous présente l'effet de 
l'afflux des capitaux étrangers notamment sous forme de dette à court terme sur la vulnérabilité du système bancaire. Toutefois, ces modèles ignorent l'existence de la monnaie et donc du taux de change nominal.

Récemment, Diamond et Rajan (2006) ont introduit la monnaie dans leur modèle d'une crise bancaire dans une petite économie fermée. L'originalité de leur modélisation est de permettre non seulement une analyse de la structure du capital bancaire, mais aussi de la politique monétaire ainsi que de la relation entre le système financier et les secteurs réels.

Les statistiques plus récentes indiquent que les capitaux étrangers jouent un rôle de plus en plus crucial dans l'investissement d'un pays, ce phénomène est particulièrement notable dans les petites économies ouvertes. Ainsi, en étendant le modèle Diamond et Rajan (2006) à une petite économie ouverte, il est possible d'expliquer la crise islandaise et celle des PECO.

Cet article consiste en une extension du modèle Diamond et Rajan (2006) dans une petite économie ouverte afin de prendre en compte les caractéristiques principales des crises financières récentes dans les PECO et en Islande. Le modèle met l'accent sur le rôle des banques dans le transfert de liquidités de l'économie, ceci étant justifié par les crises récentes dans les petits pays ouverts. En effet, l'illiquidité internationale du système bancaire est au cœur de la crise financière tant pour les pays asiatique en 1997 que pour les PECO et l'Islande actuellement.

Ce modèle d'une petite économie ouverte permet d'analyser les influences distinctes des différents moyens de financement (le capital bancaire, la dette à court terme et le dépôt), les impacts de la dénomination des passifs bancaires (en devise ou en monnaie nationale) sur la stabilité du système financier, ainsi que la connexion entre les secteurs réels, le système 
financier et la monnaie. Le modèle permet également de discuter les effets de la politique monétaire et du change dans le cas où une majorité des obligations et des revenues bancaires sont libellés en devise.

Le reste de l'article est organisé en quatre sections: la prochaine section traitera le cadre général du modèle; la section III examinera le problème de maximisation des banques commerciales et la section IV discutera la politique monétaire en cas de crise de liquidité. La dernière section résume les résultats de notre analyse.

\section{Cadre du modèle}

\section{II.1 Hypothèses de base}

Ce modèle étend celui de Diamond et Rajan (2006) en économie ouverte tout en adoptant leurs hypothèses sur la définition et la fonction de la monnaie et du bon du Trésor, ainsi que le rôle des banques commerciale dans l'économie. A la différence de leur modèle, nous adoptons en premier lieu l'hypothèse d'une petite économie ouverte aux mouvements des capitaux internationaux et des produits étrangers. Nous introduisons ainsi le taux de change nominal et la notion du ratio minimal du capital bancaire. Dans cette petite économie ouverte, les dotations initiales des agents domestiques sont supposées être relativement insuffisantes par rapport aux projets à investir. ${ }^{2}$ Les banques recourent au marché financier international dans le but de financer plus de projets que ce qu'elles peuvent financer avec les ressources financières domestiques. Ainsi, les capitaux étrangers peuvent être investis et/ou apporter des liquidités dans cette petite économie ouverte. En suivant Chang et Vélasco

\footnotetext{
${ }^{2}$ Le modèle de Diamond et Rajan (2006) consiste en une analyse de fragilité du système bancaire d'une petite économie fermée, où la seule source d'investissement est constituée par les dépôts des résidentes.
} 
(2001), nous introduisons l'emprunt étranger à court terme, représenté par $D^{*}$, dans le financement des projets domestiques à la période initiale, puis à la période intermédiaire où les banques doivent décider de restructurer ou non les projets de long terme. Par ailleurs, La notion d'IDE est intégrée dans le modèle, qui est représenté par $k^{*}{ }^{3}$ Dans ce petit pays, la taille du capital national est tellement restreinte par rapport à l'ampleur des afflux de capitaux étrangers que nous ignorons le capital domestique dans la composition du fond propre des banques. L'introduction de l'IDE permet de mieux adapter le modèle à la situation réelle des PECO. L'analyse des effets du flux des capitaux étrangers en intégrant le rôle de la monnaie, du taux de change nominal et de l'IDE se distingue de celle du modèle de Chang et Velasco (2001).

Sous l'hypothèse d'une petite économie ouverte, nous admettons que le niveau du prix national ne peut pas influencer le prix international et que ce dernier est supposé constant et égal à $1\left(P_{0}^{*}=\overline{P_{t}^{*}}=1\right)$. Ainsi, une unité de devise conserve une valeur réelle constante. De cette façon, dans cet article, tous les engagements libellés en devise sont traités comme contractés en terme réel et le taux d'intérêt impliqué est nommé le taux d'intérêt réel. Vu que la valeur de la monnaie nationale varie à travers le temps, les engagements libellés en monnaie nationale sont traités comme contractés en terme nominal et le taux d'intérêt appliqué est appelé le taux d'intérêt nominal. Il convient d'indiquer que les dettes auprès de non-résidents sont toujours libellées en devise.

\footnotetext{
${ }^{3}$ Le modèle de Chang et Vélasco (2001) adopte la notion des flux des capitaux étrangers dans l'analyse de crises financières. Cependant, ils n'ont pas donné un rôle à l'IDE ni au capital dans la composition de la structure du fonds propre bancaire. En effet, une intégration de l'IDE est cruciale à l'analyse des crises récentes, notamment dans le cas des PECO, parce que les banques de ces pays sont principalement contrôlées par les banques occidentales à travers l'IDE.
} 
Nous adoptons également l'hypothèse de la flexibilité du prix du modèle de Diamond et Rajan (2006). Etant données les quantités de la monnaie et des biens, l'ajustement du niveau du prix est le mécanisme le plus directe et efficace pour réaliser l'équilibre des marchés. Afin d'introduire la motivation des agents dans les transactions des biens, nous adoptons l'hypothèse de Diamond et Rajan (2006) que personne ne peut consommer ses propres dotations ou produits. Par ailleurs, toutes les transactions sont soumises à la contrainte de paiement préalable (payment in advance $)^{4}$. Par exemple, afin de consommer un produit ou un bien cash produit à la date $t$, l'acheteur est obligé de payer au vendeur à la date $t-1$. Quand les biens sont payés en dépôt ou en crédit bancaire, une transaction initiée à la date $t-1$ va réduire le crédit de l'acheteur à la date $t$. En recevant la monnaie ou le crédit bancaire à la date $t$, le vendeur peut initier la transaction sur l'achat du bien pour la consommation à la date $t+1$. Cette contrainte est aussi appliquée à la vente des obligations, à la restructuration et au refinancement des projets immatures.

\section{II.2 L'environnement économique}

Il s'agit d'une petite économie ouverte avec deux types de biens (les produits et les biens cash) peuplée par six types d'agents: les autorités, les marchands de biens cash, les investisseurs domestiques, les investisseurs étrangers, les entrepreneurs domestiques et les banques commerciales domestiques.

\section{II.2.1 Les biens}

Les produits sont librement échangés sur le marché mondial et peuvent être consommés ou investis. Les biens cash se situent en dehors de l'économie formelle et ne peuvent être

\footnotetext{
${ }^{4}$ Cette hypothèse est également adoptée par Diamond et Rajan (2006).
} 
taxés par le gouvernement. En plus, ils ne peuvent être vendus que contre de la monnaie nationale, alors que les produits peuvent aussi bien être achetés par la monnaie que par les crédits bancaires. Les biens cash pourraient être vendus lors de transactions de nature opportuniste ou dans celles dont le coût relatif pour établir une opération de crédit sera trop élevé. Pour fixer l'idée, supposons qu'il est le service offert par le marchand du bien cash. En vue de cacher son identité vis-à-vis de l'autorité fiscale, le marchand du bien cash n'accepte que la monnaie pour la vente du bien cash. L'utilité offerte par la consommation d'une unité de bien cash est équivalente à l'utilité offerte par une unité du produit. Ainsi, une unité du bien cash est un substitut parfait à une unité du produit. Le prix du bien cash vendu à la date 0 et délivré à la date 1 est équivalent au prix du produit à la période initiale. De même, le prix du bien cash vendu à la date 2 et délivré à la date 3 est égal au prix du produit réalisé à la date 2. Quand le pays subit un choc dans sa production nationale, les agents domestiques détenant de la monnaie nationale peuvent acheter les produits étrangers. Dans ce modèle, les agents choisissent le bien cash comme la meilleure alternative au bien étranger en cas de contraction temporaire de l'activité nationale.

\section{II.2.2 Agents, dotations, actifs financiers et la taxe}

Supposons qu'à la période initiale le petit pays ouvert possède $R$ unités de devise forte, détenues par la banque centrale comme réserve, et $e$ unités de produits, ainsi que $M_{0}$ unités de monnaie nationale et des bons du trésor nominaux. Ces derniers arrivent à maturité en $B_{2}$ unités de monnaie nationale à la date 2 . Il convient d'indiquer que la valeur des actifs nominaux à la période initiale est déterminée par la quantité des produits et de devise 
possédés par le pays. ${ }^{5}$ Enfin, tous les produits et actifs nominaux sont délivrés à la période initiale à un grand nombre d'investisseurs domestiques identiques.

Dans cette économie, seuls les entrepreneurs domestiques ont une technologie à rendement constant et non-transférable. A défaut de dotation initiale, chaque entrepreneur ayant un projet illiquide doit demander un investissement d'une unité de produit auprès d'un banquier avant la date 0 . Il y a deux types de projets dans l'économie: une proportion $\alpha$ des projets est réalisée à la date 2 et une proportion $1-\alpha$ arrive à maturité à la date 4 . Cependant, au moment d'investir, aucun agent ne peut connaître le type du projet investi et cette information sera révélée à la date 0 . Chaque unité de produit investi peut rapporter $X(>1)$ unités de produits, si le projet est fini, quelque soit la date de maturité.

Le gouvernement taxe les entrepreneurs sur la vente des produits et la taxe n'est payée qu'en monnaie nationale. Le montant nominal de la taxe sur un projet mature à la date $t$ est donc :

$$
\frac{\tau C_{t}}{1-\tau} P_{t}=\tau X_{t} P_{t}
$$

où $\tau$ représente le taux d'imposition. Supposons que pour chaque projet mature $C_{t}$ est la quantité de produits après impôt, $P_{t}$ signifie le niveau du prix domestique à la date $t$. D'ailleurs, cette taxe est appliquée également sur la restructuration des projets immatures. Pour chaque projet restructuré à la date $t$, le montant nominal de taxe est donc:

$$
\frac{\tau}{1-\tau} c P_{t}
$$

\footnotetext{
${ }^{5}$ Cette hypothèse est très importante pour déterminer la valeur de la monnaie nationale dans la période initiale ou le prix du produit avant la date 0 , ainsi que le taux de change nominal avant la date 0 et à la date 0 . Ceci est différent du modèle d'une petite économie fermée de Diamond et Rajan (2006), qui met l'accent sur l'évolution du prix après la date initiale. Cependant, leur modèle n'a pas fourni la manière de déterminer le prix du bien en monnaie nationale à la date initiale. En revanche, pour un modèle d'une économie ouverte, le niveau initial du prix est crucial, car il est le benchmark pour évaluer la valeur et la volatilité de la monnaie nationale dans le temps.
} 
Les banques se situent au cœur de l'économie et prennent le rôle d'intermédiaire des liquidités. Nous supposons que toutes les banques domestiques sont identiques et qu'elles n'ont pas de ressources propres. En se dotant d'un capital humain spécial, elles collectent toutes les dotations initiales des investisseurs domestiques, absorbent les capitaux étrangers, et les investissent ensuite dans les projets illiquides des entrepreneurs. L'habileté spéciale des banques leur permet également de collecter $\gamma C_{t}(<1)$ unités de produits auprès d'un projet arrivant à maturité, et l'entrepreneur garde la partie résiduelle i.e., $(1-\gamma) C_{t}$. Dans les périodes de contraction, en manque de liquidités, les banques peuvent aussi choisir de restructurer les projets immatures en obtenant $c(c<1)$ unité de produit. ${ }^{6}$ Nous pouvons résumer ces relations par l'inéquation (3):

$$
c<1<\gamma C<C
$$

\section{II.2.3 Taux d'intérêt}

Nous appelons les obligations contractées en devise 'les obligations en terme réel'. Le taux d'intérêt appliqué à ces obligations est 'le taux d'intérêt réel', $(r)$. Lorsque les obligations sont libellées en monnaie nationale, nous les appelons les obligations nominales. Le taux d'intérêt de celles-ci est 'le taux d'intérêt nominal' (i) Sous l'hypothèse de la libre circulation des capitaux, si les banques émettent les dépôts nominaux afin d'assurer le financement des investissements, elles doivent garantir l'égalité entre l'utilité offert par le dépôt réel et le dépôt nominal. De ce fait, la condition de parité des taux d'intérêt doit être vérifiée à l'équilibre:

\footnotetext{
${ }^{6}$ La restructuration implique la liquidation d'un projet immature afin de récupérer certaines unités de produits à la date présente. Il convient d'indiquer que cette opération peut être conduite tant par les banques que par les actionnaires. De ce fait, les actionnaires peuvent prendre le relais des banques, rembourser les créanciers et récupérer le bénéfice, seulement si ce bénéfice est plus important que le dividende offert. Par conséquent, à la date intermédiaire, le dividende distribué aux actionnaires doit au moins être égal au bénéfice attribué au capital dans le cas de désintermédiation des banques.
} 


$$
i_{i j}=r_{i j}+\frac{E_{j}-E_{i}}{E_{i}}
$$

où $i_{i j}$ et $r_{i j}$ représentent respectivement le taux d'intérêt nominal de la date $i$ à la date $j$ et le taux d'intérêt réel de la même période. Dans l'équation (4), nous admettons que les agents privés forment des anticipations parfaites.

Supposons que le taux d'intérêt réel sans risque de la première période sur le marché financier international est $1\left(r_{02}=1\right)$, nous avons $i_{02}=\frac{E_{2}}{E_{0}}$. De ce fait, nous pouvons obtenir: $i_{02} \geq r_{02}$. Cette relation donne une illusion qu'emprunter en devise semble moins cher qu'en monnaie nationale. En effet, ceci nous explique en partie la raison à laquelle les PECO ont massivement emprunté en devise forte.

Dans la deuxième période, il y n'aura plus d'incertitude sur les projets investis. Tous les projets continus seraient matures à la date 4. Cependant, les investisseurs ont une préférence pour la consommation à la date 2 qu'à la date 4 , le taux d'intérêt étranger de la deuxième période sera toujours plus grand que 1. Par ailleurs, ce taux est influencé davantage par la situation financière des créanciers des banques domestiques. Lorsqu'un choc frappe le marché financier international, sur lequel même les prêteurs des banques sont confrontés à une contraction de liquidité, ils préfèrent récupérer les investissements que de renouveler les dettes à court terme. Cette situation est traduite dans le modèle par une hausse violente du taux d'intérêt étranger de la deuxième période.

Ici le taux d'intérêt nominal $i_{i j}$ est également le taux d'intérêt appliqué aux bons nominaux de la période correspondante.

II.2.4 Prix, monnaie et taux de change 
Pour attribuer un rôle et donc de la valeur à la monnaie nationale dans ce modèle, nous avons adopté des hypothèses similaires à celles de Diamond et Rajan (2006). Afin d'adapter leurs concepts de la monnaie dans ce modèle d'une petite économie ouverte, on intègre le rôle du taux de change nominal et définit la relation entre le niveau du prix en monnaie nationale et le taux de change nominal.

La monnaie domestique est dotée de deux rôles dans l'économie. D'une part, seule la monnaie et les bons du Trésor matures peuvent être utilisés pour payer la taxe à la date future sur la transaction des produits. D'autre part, seule la monnaie exerce la fonction de moyen de transaction dans les achats des biens cash.

Le produit est librement échangé sur le marché international et il est le seul bien existant dans l'économie formelle. D'après la théorie de la parité de pouvoir d'achat, le taux de change entre la monnaie nationale et la devise doit être égal au rapport du prix du produit sur le marché du bien national à celui d'international. Par conséquent, dans la période initiale (avant la date 0 ), nous avons:

$$
E_{0}=\frac{P_{0}}{P_{0}^{*}}
$$

où $E$ représente le taux de change nominal, c'est-à-dire le prix d'une unité de monnaie étrangère exprimée en termes de monnaie domestique. Nous avons $P_{0}=E_{0}$ dans la période initiale. ${ }^{7}$ En régime de change flexible, la variation du taux de change nominal est équivalente à celle du prix national $\left(\frac{\partial P_{t}}{\partial E_{t}}=1\right)$. Pourtant, cette relation n'est pas forcément respectée dans le cas où le pays adopte un régime de change fixe.

\footnotetext{
${ }^{7}$ Rappelons que le prix international est supposé constant et égal à $1\left(P_{0}^{*}=\overline{P_{t}^{*}}=1\right)$.
} 
La valeur de la monnaie est déterminée par le pouvoir d'achat d'une unité de monnaie, c'est-à-dire qu'elle est mesurée par le niveau du prix des produits de chaque période, qui est inversement proportionnel à la valeur présente des impôts. Cette dernière représente une part constante de la production réelle actualisée à la date 2 .

$$
\begin{aligned}
& P_{12}=\frac{M_{0}+B_{2}}{\tau\left(S_{1}+\frac{S_{2}}{r_{24}}\right)}=\frac{M_{0}+B_{2}}{\tau S_{1}+\frac{M_{2}+B_{4}}{r_{24} P_{24}}}, \\
& P_{24}=\frac{M_{2}+B_{4}}{\tau S_{2}},
\end{aligned}
$$

où $S_{1}$ et $S_{2}$ représentent respectivement la quantité totale du produit offerte par les projets des entrepreneurs à la date 2 et à la date $4 .^{8} P_{02}$ et $P_{24}$ désignent respectivement le niveau du prix à la première période et à la deuxième période.

Dans l'équation (6), $M_{0}+B_{2}$ indique le niveau de la masse monétaire de la première période. Il est supposé être constant. Une partie de la monnaie est destinée à l'achat $\tau S_{1}$ unités de produits finis et consommés à la date 2 au prix $P_{02}$. Lorsque les entrepreneurs reçoivent la monnaie, ils l'utilisent pour payer l'impôt au gouvernement. De ce fait, cette partie de monnaie ne circule plus sur le marché monétaire. La liquidité restante $\left(M_{0}+B_{2}-\right.$ $\left.\tau S_{1} P_{02}\right)$ est détenue sous forme de nouvelle monnaie $M_{2}$ et de nouveaux bonds $B_{4}$ pour financer la consommation de $\tau S_{2}$ unités de produits matures à la date 4 . Ces nouveaux bons du Trésor arrivent à sa maturité à la date 4 en $B_{4}$ unités de monnaie. Supposons qu'il n’y aura pas de nouvelle monnaie et de nouveaux bons du Trésor émis après la date 2 , la quantité agrégée de la monnaie et des bons du Trésor après la date 2 reste constante.

L'équation (7) nous montre que le niveau du prix domestique de la deuxième période est déterminé par la masse monétaire $M_{2}+B_{4}$ et l'offre du produit de la même période.

\footnotetext{
8 Dans les sections suivantes, $S_{1}$ et $S_{2}$ représentent respectivement les nombres des projets dans la première période et dans la deuxième période, qui seraient être remplacés par d'autres paramètres.
} 
Comme à la date 2 , les entrepreneurs paient l'impôt avec la monnaie procurée par la vente du produit. En conséquence, après la date 4 , le gouvernement récupère toute la monnaie, il ne reste donc plus de monnaie sur le marché.

Ainsi, d'après l'équation (5), le taux de change nominal en régime de change flexible à la date 2 et à la date 4 pourrait être obtenus comme suit: $E_{2}=P_{02}$ et $E_{4}=P_{24}$. En cas de régime de change fixe, l'équation (5) n'est pas toujours satisfaite. Le taux de change nominal pourrait être toujours identique dans le temps, si les réserves de devise du pays sont suffisamment élevées pour soutenir ce taux.

\section{II.2.5 Niveau minimum du capital}

Le dépôt bancaire est un droit fixé et non-négociable. Les déposants peuvent retirer toutes leurs ressources à toutes les dates suivant un ordre séquentiel jusqu'à ce que les banques épuisent toutes leurs réserves et tous leurs actifs. Les détenteurs des dettes à court terme ont le droit de demander le remboursement seulement quand la dette est mature. Seuls les dividendes sont ajustables, puisque les actionnaires (capital) peuvent uniquement partager la valeur résiduelle avec les banques. De cette façon, le financement par le capital permet de mieux résorber les chocs négatifs et renforce le système bancaire.

Afin d'assurer la stabilité du système bancaire, les autorités du petit pays astreignent donc les banques commerciales à respecter un niveau minimal du capital, noté par $K$. Il implique que la valeur minimale du capital ne doit pas être plus petite que $K$ pourcents de la valeur totale du capital et des obligations des banques. Quand le niveau minimal du capital est respecté, les banques peuvent collecter de nouveaux capitaux à tous les temps. ${ }^{9}$

\footnotetext{
${ }^{9}$ Pour plus de détail sur la notion du niveau minimum du capital, voir Diamond et Rajan (2000 et 2001).
} 
Sachant que chaque projet demande un investissement d'une unité de produit, et que la banque peut recevoir $\gamma C$ unités de produits à la date 4 réalisés par chaque projet. Admettons que $d^{S}$ et $D_{s}^{*}$ représentent respectivement la valeur du dépôt et la dette étrangère à court terme que la banque collecte à la date 2 en contrepartie d'un projet mature à la date 4 . De ce fait, à la date 4, les créanciers bancaires prennent une valeur fixe de $d^{s}+D_{s}^{*}$ sur $\gamma C$ et le capital partage la valeur résiduelle avec la banque et prend $\frac{\gamma C-d-D^{*}}{2} \cdot{ }^{10} \mathrm{Si}$ la valeur du capital investi dans ce projet satisfait tout juste l'exigence du ratio minimum du capital, nous avons:

$$
K=\frac{\frac{1}{2}\left[\gamma C-\left(d^{s}+D_{s}^{*}\right)\right]}{\frac{1}{2}\left[\gamma C+\left(d^{s}+D_{s}^{*}\right)\right]},
$$

où le numérateur du côté droit représente la valeur du capital à la date 4 et le dénominateur est la valeur du capital plus celle des obligations de la banque à la date 4, soit le paiement total de la banque à la date 4 . Ceci implique également que le montant maximal des obligations que la banque peut collecter à la date 2 pour un projet mature à la date 4 est $\frac{\gamma C}{1+K}$. Si nous prenons en compte le taux d'intérêt réel entre la date 2 et la date $4\left(r_{24}\right)$, en respectant le niveau minimal du capital, le montant maximal alors la banque peut collecter à la date 2 auprès d'un projet mature à la date 4 devient: ${ }^{11}$

$$
\frac{\gamma C}{r_{24}(1+K)}
$$

\section{II.3 Timing}

Dans ce modèle, il y a cinq dates indexées respectivement par $t=0,1,2,3,4$. La date avant la date 0 est traitée comme la période initiale, les dates avant et à la date 2 représentent

\footnotetext{
${ }^{10}$ Voir Diamond et Rajan (2000) pour une discussion sur le partage de profit entre le capital et la banque dans le cadre d'un jeu extensive entre les banques, les actionnaires et les entrepeneurs.

${ }^{11}$ D'une manière plus générale, la valeur que les banques peuvent collecter à la date 2 ne doit pas surpasser une fraction $\frac{\gamma C}{r_{24}(1+K)}$ de la valeur des banques à la date 4 .
} 
la première période (ou le court terme) et les dates après la date 2 représentent la deuxième période (ou le long terme).

Avant la date 0 , les banques émettent les dépôts matures en date 2 en contrepartie de la monnaie $\left(M_{0}\right)$ et des bons $\left(B_{2}\right)$ collectés auprès des investisseurs domestiques (déposants). Ces actifs financiers collectés sont traités comme les réserves des banques commerciales. Sur la base de ces réserves, les banques émettent les crédits bancaires et les délivrent aux entrepreneurs. Ensuite, les entrepreneurs achètent $e$ unités des produits chez les investisseurs domestiques et les investissent dans leurs projets illiquides.

Les investisseurs domestiques, en recevant les crédits après la vente des produits, les déposent dans leurs comptes bancaires. La valeur du dépôt réel à la date 2 est: $d_{2}=r_{02} d_{0}=$ $e+\frac{M_{0}+B_{2}}{P_{0}}$, où $d_{0}$ représente la valeur des dépôts bancaires avant la date 0 et $\frac{M_{0}+B_{2}}{P_{0}}$ la valeur réelle des actifs financiers des banque à la date $0 .{ }^{12}$

Lorsque les dépôts sont contractés en terme nominal, le montant total de la monnaie nationale que les banques doivent aux déposants à la date 2 devient: $d_{2}=i_{02} d_{0}=$ $\frac{E_{2}}{E_{0}}\left(e P_{0}+M_{0}+B_{2}\right)=\frac{P_{02}}{P_{0}}\left(e P_{0}+M_{0}+B_{2}\right)$.

Par ailleurs, les banques absorbent des financements extérieurs (y compris les dettes étrangères à court terme $\left(D^{*}\right)$ et l'IDE $\left(k^{*}\right)$ et prêtent tout de suite tous les capitaux étrangers collectés aux entrepreneurs.

Enfin, la valeur totale des investissements dans les projets domestiques avant la date 0 est donnée par $e+\frac{k^{*}+\mathrm{D}^{*}}{P^{*}}$.

A la date 0 , tous les agents connaissent $\alpha$, qui représente la fraction des projets matures à

\footnotetext{
${ }^{12}$ Dans cet article, nous supposons toujours que $r_{02}=1$, donc pour la simplicité, nous ignorons le taux d'intérêt réel $r_{02}$ dans le formule pour les sections suivante.
} 
la date 2, ainsi que la quantité des produits réa lisés à la date $2 .{ }^{13}$ Les biens cash consommés à la date 1 sont vendus à la date 0 .

A la date 1 , les biens cash vendus à la date 0 sont délivrés à la date 1 . Les déposants domestiques et les prêteurs étrangers initient les transactions liées aux achats des produits consommés à la date 2 (la situation étant identique en date 3).

Cependant, quand il y a un manque de produits sur le marché du bien domestique et qu'une partie de la demande de produits exprimée par les créanciers ne peut être satisfaite, les créanciers vont soit demander aux banques de rembourser leurs créances matures à la date 2, soit renouveler leurs prêts aux banques. En même temps, les banques commencent à collecter les nouveaux fonds ou à restructurer les projets immatures en cas de manque de liquidité à cause de la contraction de la production.

A la date 2, les entrepreneurs des projets matures à la date 2 remboursent les dettes et paient la taxe avec la monnaie. Le gouvernement rachète les bons matures et émet de nouveaux bons et de la nouvelle monnaie. Les banques remboursent les obligations et partagent la valeur résiduelle avec le capital (les actionnaires).

A la date 3, les entrepreneurs ayant des projets matures à la date 4 initient les transactions sur les ventes de ces produits.

A la date 4, le gouvernement rachète les bons du Trésor matures. Les entrepreneurs remboursent les banques. Ces dernières remboursent les obligations et récompensent les actionnaires. Les banquiers, les entrepreneurs et tous les investisseurs de la $2^{\text {ème }}$ période consomment.

${ }^{13}$ Le paramètre $\alpha$ représente le niveau du décalage entre la maturité de passifs bancaires et celles des actifs. 


\section{Le problème de maximisation des banques à la date 2}

Dans cet article, nous faisons une attention particulaire aux impacts des facteurs de crise d'origine internationale sur la situation financière d'un petit pays. Ces facteurs affectent le petit pays notamment via les flux des capitaux étrangers et les coûts des emprunts étrangers.

En vue d'honorer leur obligations et de maximiser leur profit, les banques cherchent à maximiser leur valeur réelle à la date 2 . Le problème de maximisation des banques à cette date est donc:

$$
\max _{\mu}\left\{\frac{M_{0}+B_{2}}{p_{12}}+\left(e+\frac{k^{*}+D_{1}{ }^{*}}{P^{*}}\right) \alpha \gamma C+\left(e+\frac{k^{*}+D_{1}^{*}}{P^{*}}\right)(1-\alpha) \mu c+\left(e+\frac{k^{*}+D_{1}{ }^{*}}{P^{*}}\right)(1-\mu)(1-\alpha) \frac{\gamma C}{r_{24}}\right\} .
$$

En effet, la somme des termes dans (10) représente la valeur réelle des banques à la date 2 . Le première terme de (10) décrit la valeur réelle des actifs financiers détenus par les banques, le deuxième terme est le montant réel que les projets matures permettent de collecter à la date 2 , le troisième terme indique la valeur récupérée grâce à la liquidation d'une fraction $\mu$ $(0 \leq \mu \leq 1)$ des projets immatures et le dernier terme implique la valeur actualisée à la date 2 que les banques pourraient obtenir en laissant des projets continuer jusqu'à la maturité à la date 4 .

Dans ce modèle d'économie ouverte, afin d'assurer l'équilibre général, le problème de maximisation des banques doit satisfaire deux conditions: l'équilibre sur le marché monétaire et l'équilibre sur le marché des biens. Ici, la condition d'équilibre sur le marché des biens est équivalente à celle de la contrainte de solvabilité bancaire, du fait que les banques prennent le rôle de transfert de liquidité dans l'économie. Ainsi, tous les investissements dans des projets et toutes les transactions du produit sont réalisées à travers les banques. Par conséquent, quand la condition de solvabilité bancaire est satisfaite, la 
condition d'équilibre sur le marché du produit est réalisée également.

La contrainte de solvabilité des banques à la date 2 est donc:

$$
\left\{\begin{array}{l}
V\left(r_{24}, p_{12}, \mu\right) \geq \underset{\mu^{C}}{ } \frac{\operatorname{Max}}{2}\left\{V\left(r_{24}, p_{12}, \mu\right)+r_{02}\left(\frac{E_{2} D_{1}{ }^{*}}{P^{*}}+d_{0}\right)\right\} \\
\operatorname{avec} V\left(r_{24}, p_{12}, \mu\right)= \\
\quad \frac{M_{0}+B_{2}}{p_{12}}+\left(e+\frac{k^{*}+D_{1}{ }^{*}}{P^{*}}\right) \alpha \gamma C+\left(e+\frac{k^{*}+D_{1}{ }^{*}}{P^{*}}\right)(1-\alpha) \mu c+\left(e+\frac{k^{*}+D_{1}{ }^{*}}{P^{*}}\right)(1-\mu)(1-\alpha) \frac{\gamma C}{(1+k) r_{24}},
\end{array}\right.
$$

où, le côté droit de l'inéquation (11) décrit la demande totale de liquidité du capital et des créanciers des banques à la date 2 . Le côté gauche indique la valeur maximale que les banques peuvent posséder à la date 2 ou l'offre maximale de produits à cette date. Ceci est composé des actifs financiers des banques, des valeurs collectées auprès des entrepreneurs ayant des projets matures à la date 2, du montant réel récupéré par la restructuration des projets immatures, ainsi que de la valeur que les banques peuvent collecter à la date 2 auprès des projets matures à la date 4 .

La condition équilibre sur le marché monétaire à la date 2 est décrite par

$$
\frac{M_{0}+B_{2}}{p_{12}}=\frac{t}{1-t}\left(e+\frac{k^{*}+D_{1}^{*}}{P^{*}}\right)\left[\alpha C+(1-\alpha) \mu c+\frac{(1-\mu)(1-\alpha) C}{r_{24}}\right],
$$

où les trois termes dans le crochet représentent la valeur totale des produits offerte par les projets investis dans les deux périodes actualisée à la date 2. En effet, l'équation (12) est équivalente à l'équation (6). Nous savons déjà que toute la monnaie sera dépensée pour le paiement de la taxe à la fin de la date 4. Si l'équation (6) est satisfaite, il n'y aura plus de monnaie sur le marché monétaire à la fin de la date 4. Par conséquent, l'équation (12) est également la condition d'équilibre sur le marché monétaire.

Rappelons que $\alpha$ représente la fraction des projets matures à la date 2. Etant donné le montant des passifs bancaires à court terme, $\alpha$ désigne également le degré de décalage entre 
la maturité des passifs et celle des actifs des banques. D'après les deux conditions d'équilibres, si $\alpha$ est assez grand de tel sort que la condition $\frac{M_{0}+B_{2}}{P_{12}}+\left(e+\frac{k^{*}+D_{1}^{*}}{P^{*}}\right) \alpha \gamma C \geq$ $r_{02}\left(\frac{E_{2} D_{1}^{*}}{P^{*}}+d_{0}\right)$ est satisfaite, les actifs bancaires sont en toute sécurité et aucun projet sera restructuré. Ainsi, l'influence des chocs étrangers est minimale voire nulle sur les investissements des banques.

Toutefois, lorsque les actifs des banques sont principalement concentrés sur les projets à long terme, les passifs à court terme peuvent menacer la stabilité du secteur bancaire. On constate qu'il y aura un risque de ruée bancaire, si $\frac{M_{0}+B_{2}}{P_{12}}+\left(e+\frac{k^{*}+D_{1}^{*}}{P^{*}}\right) \alpha \gamma C+(e+$ $\left.\frac{k^{*}+D_{1}^{*}}{P^{*}}\right)(1-\alpha) c<r_{02}\left(\frac{E_{2} D_{1}^{*}}{P^{*}}+d_{0}\right)$. C'est-à-dire que même si les banques restructurent tous les projets immatures à la date 2, il y aura encore des obligations non remboursées. Dans ce cas-là, les banques seront solvables seulement si elles peuvent renouveler leurs dettes à court terme à un coût raisonnable. Autrement dit, le taux d'intérêt réel étranger de la deuxième période $\left(r_{24}\right)$ doit être au niveau faible de telle sorte que

$$
\left(e+\frac{k^{*}+D_{1}^{*}}{P^{*}}\right)(1-\alpha) \frac{\gamma C}{(1+K) r_{24}} \geq r_{02}\left(\frac{E_{2} D_{1}^{*}}{P^{*}}+d_{0}\right)-\frac{M_{0}+B_{2}}{P_{12}}+\left(e+\frac{k^{*}+D_{1}^{*}}{P^{*}}\right) \alpha \gamma C .
$$

Par conséquent, le système bancaire est très vulnérable face aux chocs sur le marché financier international. Si ce dernier est frappé par des chocs négatifs, le coût d'emprunt des petits pays grimpe rapidement jusqu'à ce que les banques des petits pays ne puissent plus supporter. Suite à la crise des subprimes aux Etats-Unis en 2007 et en raison de ses répercussions sur le marché financier international, beaucoup de banques des petites économies sont confrontées à une situation d'illiquidité voire d'effondrement total du système financier. Nous observons que les petites économies, telles que l'Islande et les PECO, s'enfoncent dans une crise d'illiquidité internationale: Soit leurs accès sur le marché 
financier international est coupé totalement, soit le niveau du taux d'intérêt des emprunts étrangers_monte très violement.

Dans les cas intermédiaires, les banques ne seront pas mises en faillite, néanmoins, le problème de maximisation des banques à la date 2 , ou le choix des banques entre collecter de nouveaux fonds et restructurer les projets immatures dépend principalement du niveau du taux d'intérêt international de la deuxième période $\left(r_{24}\right)$.

Lorsque le taux d'intérêt réel étrangers de la deuxième période est tel que $r_{24} \leq \frac{\gamma C}{c}$, les banques peuvent obtenir plus de bénéfices en laissant continuer des projets immatures. En fait, $\frac{\gamma C}{c}$ signifie la récompense des banques en renonçant à la restructuration des projets à la date 2, et il est également le niveau de taux d'intérêt réel, le plus élevé que les banques peuvent prévoir. Ainsi, pour $r_{24}=\frac{\gamma C}{c}$, les banques sont indifférentes entre les deux options.

Par ailleurs, dans le cas où $r_{24} \leq \frac{\gamma C}{c}$ il existe encore deux sous situations: $r_{24} \leq \frac{\gamma C}{(1-K)_{c}}$ et $\frac{\gamma C}{(1-K)_{c}}<r_{24} \leq \frac{\gamma C}{c}$. Lorsque $r_{24 \leq \frac{\gamma C}{(1-K) c}}$, il est certain qu'aucun projet immature ne sera restructuré. Tandis que si $\frac{\gamma C}{(1-K)_{c}}<r_{24} \leq \frac{\gamma C}{c}$, le montant réel de nouveaux fonds est susceptible d'être moins important que celui obtenu en restructurant tous les projets immatures $(c)$. Quand les banques sont face au manque de liquidité très important, elles sont obligées de restructurer une fraction minimale des projets de sorte à satisfaire la demande de liquidité à la date 2 .

Dans le cas où le taux d'intérêt réel de la deuxième période sur le marché financier international est trop élevé $\left(r_{24}>\frac{\gamma c}{c}\right)$, les banques sont obligées de restructurer tous les projets immatures. Puisque la valeur offerte par un projet continué et mature à la date 4 est inférieure à celle offerte par la restructuration à la date 2 . 
En effet, un taux d'intérêt trop élevé entraîne une contraction du crédit dans l'économie, qui à son tour entraîne une baisse de l'activité. Cela a pour effet de diminuer les recettes fiscales et d'augmenter le niveau du prix domestique à la date 2. La monnaie nationale se déprécie également sur le marché des changes.

Nous avons supposé que le niveau du taux d'intérêt international est un élément exogène pour le petit pays. La dégradation des conditions de liquidité dans les pays créditeurs jouent un rôle important dans le déclenchement de la crise de liquidité dans les petits pays. En effet, dans la crise financière internationale 2007-2009, lorsque la condition de liquidité des pays créditeurs du pays se détériore, les créditeurs étrangers sont pressés de récupérer leurs investissements dans ce pays pour satisfaire la demande de liquidité de leur propre économie. Par ailleurs, dans une conjoncture de crise financière internationale et d'une situation de raréfaction du crédit sur le marché financier international, les investisseurs deviennent plus prudents que dans les périodes de croissance. Tout ceci peut se traduire par une hausse du taux d'intérêt international, mais aussi par un phénomène de fuite vers la qualité ('fly to quality'). Dans la crise actuelle, nous constatons que l'accès au marché financier international des petits pays est coupé brutalement, de sorte que les banques domestiques en cas de raréfaction de liquidité causée par l'exode massive du capital étranger sont obligées de restructurer les projets immatures.

\section{Politique Economique}

Après avoir vu le mécanisme de crise bancaire, nous discuterons dans cette section la politique appropriée pour faire face à la situation d'illiquidité du système bancaire 
domestique.

\section{IV.1 L’intervention sur la restructuration des projets}

Rappelons que les banques sont indifférentes face à la restructuration ou non des projets immature, si le coût de renouvèlement des dettes à court terme est: $r_{24}=\frac{\gamma C}{c}$. On étudie, à travers l'exemple suivant, les différentes valeurs obtenues respectivement par les banques à la date 2 via la restructuration et la continuation des projets immatures pour $r_{24}=\frac{\gamma C}{c}$.

Exemple numérique: Admettons que $R=5, \tau=17 \%, C=1.5, \gamma=0.8, c=0.6, e=15$, $M_{0}=10, B_{2}=10, K^{*}=20, D_{1}{ }^{*}=65$ et $r_{02}=1$. Donc $d_{0}=35$. Si $\alpha=0.5$ et $r_{24}=\gamma C=2$, il n’y aura pas de ruée bancaire. ${ }^{14}$ Pourtant, l'offre de liquidité reste inférieure à la demande, les banques devront soit refinancer les projets immatures, soit restructurer ces projets. En cas de restructuration, le niveau du prix domestique à la date 2 est $P_{12}=0.95$ et le profit total en termes de valeur à la date 2 égal à 5.5. En cas de continuation des projets, le niveau du prix domestique à la date 2 devient plus faible et il est égal à $P_{12}=0.89$, et le profit total en termes de valeur à la date 2 égal à $6.25 .^{15}$

Quoique du point de vue des banques, la restructuration et la continuation des projets immature donnent une valeur équivalente, la continuation des projets atténue la volatilité du niveau du prix et augmente le profit des banques. Il convient de noter qu'il y a un grand nombre des banques identiques mais non-monopolistes dans cette petite économie, les banques sont donc 'les price takers'. Dans ces conditions, quand les banques choisissent entre la continuation et la restructuration des projets immatures, elles ne peuvent pas prendre en compte les impacts de leur action individuelle sur le niveau du prix et donc sur la valeur

\footnotetext{
${ }^{14}$ Il y aura la possibilité de ruée bancaire, si $\alpha<0.359$.

15 Pour le calcul intermédiaire, voir Appendice A.
} 
leurs actifs financiers. En effet, la continuation des projets fournit plus de biens au pays, augmente le montant réel de l'impôt, ainsi que la valeur des actifs nominaux. Dans un tel cas, l'intervention de l'autorité économique visant à décourager la restructuration devient cruciale non seulement pour stabiliser le système financier et la valeur de la monnaie nationale, mais aussi pour soutenir les secteurs réels du pays.

\section{IV.2. le ratio minimal du capital}

D'après le problème de maximisation des banques à la date 2 , plus le niveau du ratio minimal du capital à la période initiale est élevé, moins sera important le montant de passifs à rembourser à la date intermédiaire. Le capital bancaire peut renforcer la stabilité du système bancaire dans ce cas-là. Pourtant, l'effet du même ratio à la période de crise n'est pas toujours favorable.

Exemple numérique: Maintenant, supposons $\alpha=0.3, r_{24}=1.8<\gamma C$ et le ratio minimal du capital exigé par la banque central est $K=0.2$. Ici les banques sont en faillite, la valeur maximale qu'elles peuvent obtenir à la date 2 en recueillant de nouveaux fonds est seulement 98.6. ${ }^{16}$ Ceci est insuffisant pour rembourser les obligations matures à cette date. En conséquence, la ruée bancaire aura lieu dès la date 0 et tous les projets seront restructurés dès la date $0, \mathrm{y}$ compris les projets matures à la date 2 et le montant réel obtenu grâce à la restructuration sera seulement de $80 .{ }^{17}$ Cette situation est socialement inefficace, du fait qu'au moins une partie de la restructuration ne soit pas nécessaire. ${ }^{18}$ Par ailleurs, le niveau

\footnotetext{
${ }^{16}$ Rappelons que le montant maximal donc les banques peuvent collecter à la date 2 est seulement une fraction $\frac{\gamma C}{(1-K) r_{24}}$ de leurs valeurs à la date 4 .

${ }^{17}$ Pour le calcul intermédiaire, voir Appendice C.

${ }^{18}$ La nature de l'ordre séquentiel des retraits des dépôts détermine que les déposants arrivent plus tardivement aux banques que les autres ont des risques de ne pas être payés. Admettons qu'il y a un grand nombre de prêteurs étrangers dans la première période. En temps normal, les prêteurs ne peuvent demander le paiement avant l'échéance des dettes. Cependant, en cas de ruée bancaire, les prêteurs comme les déposants vont
} 
du prix de la première période est de $P_{12}=1.67$ et la monnaie domestique se déprécie également de $67 \% .^{19}$

Face à cette situation, si l'autorité économique baisse le niveau minimal du capital à $K=0.155$, les banques ne seront pas en faillite et aucun projet immature sera restructuré. Tous les créanciers de la première période seront payés au montant préfixé, les banques et les actionnaires partageront la valeur résiduelle à la date 4 , où chacun reçoit un bénéfice identique de 2.9 en termes de valeur à la date 2. La valeur de la monnaie reste stable avec une baisse légère du niveau du prix domestique à la date $2\left(P_{12}=0.98\right) .{ }^{20}$

Il convient d'indiquer que la crise étudiée dans cet article est principalement causé par le décalage de maturité des actifs et des passifs des banques, autrement dit que les investissements des banques sont généralement sains et qu'on préfère appelle celle-ci la crise d'illiquidité que la crise de solvabilité.

L'exigence du niveau minimal du capital a pour effet de stabiliser le système bancaire. Cependant, en période de crise, une hausse du ratio minimal du capital nuit en même temps à la santé financière des banques confrontant à la situation d'illiquidité. En conséquence, lorsque la banque centrale pratique la politique du ratio minimal du capital, elle ferait mieux de distinguer les types de problème que les banques commerciales rencontrent. La politique consiste à imposer ce ratio aux banques se trouvant dans la crise de l'illiqudité ne peut pas renforcer le système bancaire, mais au contraire entraîne une crise bancaire, ainsi qu'une

demander le remboursement dès la date 0 (au moment où ils ont appris la fraction des projets qui arrivent à la maturité à la date 2 .

${ }_{19}$ Du fait qu'il n'y aura pas de produit sur le marché du bien domestique à la date 2, lorsque tous les projets sont restructurés à la date 0 , la monnaie ne sera pas acceptée pour acheter les biens cash. Ceci s'explique par le fait que les marchands du bien cash ne prévoient pas de transaction utilisant la monnaie. Par conséquent, toute la monnaie domestique est dépensée dès la date 0 pour les produits provenant de la restructuration et la valeur de la monnaie nationale est donc déterminée par le niveau du prix à la date 0 .

${ }^{20}$ Pour le calcul intermédiaire, voir Appendice B. 
restructuration massive des projets illiquides. Une baisse de ce ratio a pour effet d'améliorer la condition de solvabilité des banques confrontées à la contraction de liquidité. Par ailleurs, une injection du fonds par l'autorité économique peut également sauver les banques qui font face au manque de liquidité temporaire.

\section{IV.3 Le rôle de la Banque Centrale comme le prêteur en dernier ressort}

En tant que prêteur en dernier ressort, la banque centrale protège son système financier en cas de crise d'illiquidité. Dans les exemples précédents, la réserve en devise de la banque centrale est supposée être $R=5$. Ce montant est suffisant pour combler le manque de liquidité des banques commerciales. Dans ce cas-là, le rôle du prêteur en dernier ressort de la banque centrale fonctionne. Pourtant, il convient d'indiquer que la capacité de la banque centrale à sauver les banques commerciales est surtout limitée par son niveau de réserve en devise. ${ }^{21}$ Dans la réalité des petites économies, nous ne pouvons trouver que rarement des petits pays satisfaisant cette exigence de réserves comme dans les exemples. Bien que les pays d'Asie du Sud-est soient relativement riches en réserves de change, leurs réserves de devise étaient seulement en moyenne de $15 \%$ du PIB en 1997. Les monnaies nationales de ces pays ont subi une forte dépréciation pendant la crise financière de 1997. Quant à l'Islande et les PECO, les réserves de change sont loin d'être suffisantes pour protéger leur système financier. Dans le cas de l'Islande, la banque centrale n'a pas trouvé une solution efficace face à l'exode soudain des capitaux étrangers. Les banques commerciales de ces pays sont forcées de restructurer quasiment tous leurs investissements à long terme. Un

\footnotetext{
${ }^{21}$ Dans cette section, toutes les obligations des banques commerciales sont contractées en termes réels (libellées en devise). Etant donné le niveau d'offre du produit sur le marché du bien domestique, une injection de la monnaie nationale par la banque centrale dans le système bancaire ne se traduit que par une hausse du niveau du prix mesuré en monnaie nationale. En conséquence, la pratique de la politique du prêteur en dernier ressort de la banque centrale doit se limiter à son niveau de réserve en devise. Ceci est vraiment le cas pour les petites économies où les contrats sont libellés en devises (dollars ou euros).
} 
effondrement du système bancaire devient donc inévitable, la monnaie du pays ayant déprécié brutalement.

\section{IV.4 Politique de 'l'open market'en cas du dépôt nominal}

A la différence du dépôt réel, le dépôt nominal ne promet qu'une somme fixe de la monnaie nationale aux déposants. Cependant sous l'hypothèse de mobilité parfaite du capital, la condition de parité des taux d'intérêt doit être vérifiée à l'équilibre afin d'assurer que les déposants consentissent à posséder le dépôt nominal. Ainsi, la valeur réelle du dépôt nominal et le problème de maximisation des banques à la date 2 ne sont pas modifiés par l'introduction du dépôt nominal par rapport au cas du dépôt réel.

Rappelons que l'utilité offerte par la consommation d'une unité de bien cash est équivalente à l'utilité offerte par une unité du produit. Ainsi, une unité du bien cash est un substitut parfait à une unité du produit. Face à une baisse de l'activité et donc face à une dépréciation de la monnaie nationale, les banques sont obligées d'augmenter le niveau du taux d'intérêt nominal afin d'éviter que tous les dépôts soient retirés pour les achats des biens cash.

Vu que les biens cash se situent en dehors de l'économie formelle, nous avons supposé que son prix pour les achats initiés à la date 0 est toujours identique au prix du produit avant la date 0 pour une quantité fixe $\left(M_{0}\right.$ unités $)$ de monnaie disponible à la date $0 .{ }^{22}$ Cependant, si la quantité de la monnaie disponible augmente $\left(M_{0}+\Delta=B_{2}-\Delta\right)^{23}$ à la date 0 à travers

\footnotetext{
${ }^{22}$ Les bons du Trésor sont matures à la date 2 , si les déposants veulent les dépenser pour les achats du bien cash à la date 0 , ils ne peuvent qu'obtenir $\frac{B_{02}}{i_{02}}$ unités de monnaie nationale. Par conséquent, les déposants n'ont aucun intérêt de les retirer avant la date 2 .

${ }^{23}$ Où $\Delta$ représente le niveau de cette opération de 'l'open market', ce qui doit être assez petit. Une ampleur très importante de cette politique expansionniste pourrait entrainer une forte volatilité de la valeur de la monnaie nationale à la date 0 .
} 
l'opération de 'l'open market', le prix du bien cash à la date 0 devrait monter simultanément. ${ }^{24}$ Cette hausse du prix à la date 0 engendre deux effets sur le système bancaire. D'un côté, elle stabilise le taux d'intérêt nominal ; d'un autre côté, elle augmente le montant qui pourrait être retiré par les déposants à la date $0 .^{25}$ Si nous revisitons la condition de solvabilité bancaire, nous savons donc: $M_{0} P_{01}+\frac{M_{0}+B_{2}}{p_{12}}+\left(e+\frac{k^{*}+D_{1}^{*}}{P^{*}}\right) \alpha \gamma C+\left(e+\frac{k^{*}+D_{1}^{*}}{P^{*}}\right)(1-$ $\alpha)_{c} \geq r_{02} \frac{E_{2} D_{1}^{*}}{P^{*}}+i_{02}\left(e P_{0}+M_{0}+B_{2}-M_{0}\right)$. En simplifiant, on obtient une nouvelle condition de solvabilité des banques:

$$
M_{0} P_{01}+\frac{M_{0}+B_{2}}{p_{12}}+\left(e+\frac{k^{*}+D_{1}{ }^{*}}{P^{*}}\right) \alpha \gamma C+\left(e+\frac{k^{*}+D_{1}{ }^{*}}{P^{*}}\right)(1-\alpha) c \geq r_{02} \frac{E_{2} D_{1}{ }^{*}}{P^{*}}+\frac{\left(e P_{0}+M_{0}+B_{2}\right)}{P_{01}},
$$

où, le premier terme du côté gauche représente la valeur réelle des biens cash achetés par les déposants à la date 0 et consommés à la date 1 , et le dernier terme du côté droit signifie la valeur réelle des dépôts à la date 2 . Si nous comparons maintenant cette condition de solvabilité avec celle en cas du dépôt réel (l'inéquation 12), nous pouvons constater que la situation financière est effectivement améliorée suite à une politique de l'open market qui consiste à acheter les bons du Trésor à la date 0 .

Il convient d'indiquer que cette politique monétaire expansionniste ne changera pas le niveau de la masse monétaire à la date 2 , ni la valeur de la monnaie nationale à cette date. En effet, un retrait du dépôt avant l'échéance sous forme de monnaie nationale entraîne simplement une baisse du niveau de réserves des banques commerciales et aucune banque n'est en faillite à cause de ce retrait.

\footnotetext{
${ }^{24}$ L'opération de 'l'open market' a lieu à la date 0 où tous les contrats initiaux sont déjà négociés et signés. Par conséquent, cette politique monétaire expansionniste ne changera pas d'autres variables déterminées avant la date 0 .

${ }^{25}$ En envisageant une dépréciation de la monnaie nationale, les banques commerciales sont obligées de réajuster à la date 0 le taux d'intérêt offert au dépôt nominal. Ce dernier doit monter pour satisfaire la condition $i_{02}=\frac{P_{12}}{P_{01}} \leftrightarrow \frac{\partial i_{02}}{\partial P_{01}}<0$. Quand le niveau du prix à la date 0 monte, les banques pourraient augmenter moins (voire rien) le taux d'intérêt nominal.
} 
Néanmoins, l'effet de cette politique monétaire reste très limité pour un pays largement endetté en devise, puisque la valeur des obligations libellées en devise n'est pas affectée par la variation de la valeur de la monnaie nationale.

Lorsque les dépôts sont contractés en monnaie nationale, la politique du prêteur en dernier ressort devient légèrement plus efficace qu'en cas du dépôt réel. En cas d'une dépréciation de la monnaie nationale, la banque centrale peut toujours garantir les passifs nominaux des banques. Par contre, pour les pays massivement endettés en devise, les effets positifs de cette politique sont donc très faibles.

En effet, dans la pratique, les pays en voie de développement (ou émergents) et les petits pays développés contractent rarement des dettes auprès des non-résidents en monnaie domestique. Les emprunts étrangers sont principalement libellés en devises dominantes. Ce phénomène observé dans des pays en voie de développement et des petits pays ouverts caractérisés par une croissance économique forte et la fragilité de leur système bancaire est appelé par Eichengreen et al. (2005) 'le péché originel'.

\section{Conclusion}

Dans un modèle d'une petite économie ouverte, caractérisée par une libéralisation financière importante qui entraîne un afflux massif des investissements directs étrangers (IDE) dans le système financier, nous examinons une crise financière causée principalement par un décalage entre la maturité des obligations et celle des projets investis ainsi que les implications en termes de stabilité du taux de change et de politique monétaire pour les pays comme Islande et les PECO. 
Nous avons montré que le financement par capital (IDE) permet d'améliorer la solvabilité du secteur financier et donc la stabilité du système bancaire du pays et réduit le risque de ruée bancaire par rapport au cas où les dettes contractés à court terme et/ou libellées en devise et les banques investissant principalement des projets à long terme. Cependant, face à la crise d'illiquidité du secteur financier, le système bancaire d'un pays est très vulnérable vis-à-vis du marché financier international. Une hausse du coût d'emprunt sur le marché international au-delà d'un certain seuil, qui dépend de la structure du modèle, devient fatale pour la solvabilité du système financier du pays.

Dans le modèle, lorsque le taux d'intérêt étranger monte au delà d'un certain seuil, le pays n'a plus de capacité de renouveler leur obligation à court terme. Pour le coût d'emprunt arrive juste à ce seuil, les banques sont indifférentes de continuer ou pas leur investissement illiquide. Pourtant, le modèle montre qu'avec ce niveau du taux d'intérêt, la continuation des projets est plus bénéfique que la restructuration. La continuation des projets peut non seulement soutenir la valeur des actifs nominaux du pays, le niveau de l'impôt, mais aussi l'activité du pays. Du fait que les banques décrites dans cette article sont les 'price takers', elles ne peuvent pas prendre en compte ces effets ci-dessus résultant de la stabilisation du niveau du prix.

La liquidation des projets immatures dans un contexte de crise de liquidité n'est pas toujours optimale du point de vue social. En effet, pour un certain niveau du taux d'intérêt intermédiaire atteint sur le marché financier international, les banques décident de liquider les projets immatures sans prendre en compte l'impact de leur action individuelle sur le niveau de prix et donc sur la valeur des actifs. Par conséquent, l'autorité économique doit 
s'employer à inciter les banques à continuer leurs projets immatures et donc à éviter une restructuration massive.

Nous avons considéré dans ce cadre les effets d'un relèvement du ratio minimal du capital destiné à rassurer les épargnants ne sont pas favorables dans la période de crise illiquidité. Cette politique a pour conséquence de limiter le montant dont les banques peuvent emprunter et elle est plus appropriée dans les périodes normales ou de croissance. Par contre, en période de crise, elle n'est inadaptée. Au contraire, une baisse de ce ratio dans une situation d'illiquidité internationale peut protéger le système bancaire et réduire le niveau de restructuration, permettant ainsi soutenir l'activité du pays, et la valeur des actifs financiers et de la monnaie nationale.

Nous avons montré qu'en régime de change flexible, une politique de 'open market' qui consiste à acheter les bons du Trésor a pour effet de soulager le fardeau des dettes des banques. Néanmoins, cette politique fonctionne seulement que si les obligations sont libellées en monnaie nationale. Ainsi, pour un pays plus ou moins dollarisé ou euroisé, la politique monétaire nationale perd partiellement voire totalement son efficacité. De même, le rôle du 'prêteur de dernier ressort' de la banque centrale est limité par son niveau du réserves en devise.

En somme, la crise décrit dans cet article n'est pas du type nouveau, pourtant, elle se répète sans cesse. La crise la plus récente à Dubaï possède les caractéristiques similaires de ceux indiqués dans le modèle. Une politique économique plus prudente pratiquée dans les périodes normales pourrait améliorer la stabilité de son système financier. Néanmoins, comme ce que le modèle a montré, la bonne politique en temps de crise de liquidité doit 
avoir pour objective de soulager le fardeau des dettes du système bancaire. Le réforme du système bancaire pourrait se dérouler en période normale où les banques sont sorties de la situation de illiquidité, afin d'éviter une restructuration massive des projets et donc un effondrement total de l'activité du pays face à la crise d'illiquidité.

\section{Appendice A}

Pour $\alpha=0.5$ et $r_{24}=2$, si les banques décident de restructurer les projets immatures, la valeur réelle des actifs financiers est: $\frac{M_{0}+B_{2}}{p_{12}}=\frac{t}{1-t}\left(e+\frac{k^{*}+D_{1}{ }^{*}}{P^{*}}\right)\left[\alpha C+\frac{(1-\alpha) C}{r_{24}}\right] \rightarrow \frac{20}{P_{12}}=0.2 *$ $100[0.5 * 1.5+0.5 * 0.6]=21$, et le niveau du prix à la date 2 est $P_{12}=0.95$. La valeur réelle des banques à la date 2 est $\frac{M_{0}+B_{2}}{p_{12}}+\left(e+\frac{k^{*}+D_{1}{ }^{*}}{P^{*}}\right) \alpha \gamma C+\left(e+\frac{k^{*}+D_{1}{ }^{*}}{P^{*}}\right)(1-\alpha) c=21+60+30=$ $111>\left(\frac{E_{2} D_{1}^{*}}{P^{*}}+d_{0}\right)=100$. Après avoir remboursé les créanciers, les banques partagent la valeur résiduelle, chacun obtenant un bénéfice de $\frac{1}{2}\left[V\left(r_{24}, \mu^{c}, p_{12}\right)-\left(\frac{E_{2} D_{1}^{*}}{P^{*}}+d_{0}\right)\right]=$ $\frac{1}{2}(111-100)=5.5$ en terme de valeur à la date 2 .

Lorsque les banques choisissent de collecter les nouveaux fonds afin de financer des projets matures à la date 4 , la valeur réelle des actifs financiers est: $\frac{M_{0}+B_{2}}{p_{12}}=\frac{t}{1-t}(e+$ $\left.\frac{k^{*}+D_{1}^{*}}{P^{*}}\right)\left[\alpha C+\frac{(1-\alpha) C}{r_{24}}\right] \rightarrow \frac{20}{P_{12}}=0.2 * 100\left[1.5 * 0.5+0.5 * \frac{1.5}{2}\right]=22.5$ et le niveau du prix à la date 2 est $P_{12}=0.89$. Chaque projet mature à la date 4 permet aux banques de collecter $\frac{\gamma C}{r_{24}(1+K)}=0.5$. Ainsi, la valeur maximale des ressources financières à la disposition des banques à la date 2 est $\frac{M_{0}+B_{2}}{p_{12}}+\left(e+\frac{k^{*}+D_{1}^{*}}{P^{*}}\right) \alpha \gamma C+0.5\left(e+\frac{k^{*}+D_{1}^{*}}{P^{*}}\right)(1-\alpha)=107.5$ et la valeur résiduelle à la date 2 est donc de 7.5. A la date 4, tous les projets sont matures, les banques collectent un montant $\left(e+\frac{k^{*}+D_{1}{ }^{*}}{P^{*}}\right)(1-\alpha) \frac{\gamma C}{r_{24}}=30$ en termes de valeur à la date 2. Après le remboursement des investisseurs de la deuxième période, la valeur résiduelle à la date 4 actualisé à la valeur à la date 2 est de $\left(e+\frac{k^{*}+D_{1}{ }^{*}}{P^{*}}\right)(1-\alpha) \frac{\gamma C}{r_{24}}-\left(e+\frac{k^{*}+D_{1}{ }^{*}}{P^{*}}\right)(1-\alpha) \frac{\gamma C}{r_{24}(1+K)}=5$. Par conséquent, la valeur 
résiduelle des deux périodes en termes de valeur à la date 2 est de 12.5 , le bénéfice des banques et celui des actionnaires sont égaux à 6.25 .

\section{Appendice B}

Si $K=0.155$, chaque projet mature à la date 4 permet aux banques de collecter $\frac{\gamma C}{r_{24}(1+K)}=$ 0.577. La valeur maximale des banques à la date 2 est $62.5+0.577\left(e+\frac{k^{*}+D_{1}{ }^{*}}{P^{*}}\right)(1-\alpha)=100$. Ce montant permet aux banques de rembourser tous les passifs matures à la date 2 et les banques ne sont pas en faillite. A la date 4 , les banques collectent un montant actualisé à la valeur à la date 2 qui est: $(1-\alpha)\left(e+\frac{k^{*}+D_{1}{ }^{*}}{P^{*}}\right) \frac{\gamma C}{r_{24}}=43.3$. On a 65 projets matures à la date 4 , qui récompensent les investisseurs de la deuxième période. La valeur résiduelle actualisée à la date 2 obtenue par les banquiers et celle obtenue par les actionnaires (le capital) sont identique, soit égales à $\frac{1}{2}\left(43.3-\frac{65 \gamma C}{r_{24}(1+K)}\right)=2.9$.

\section{Références:}

Buiter, Willem and Anne Sibert (2009), 'The collapse of Iceland's banks: the predictable end of a non-viable business model', in The First Global Financial Crisis of the 21st Century, pp. 23-26.

Calomiris, Charles W. and Charles M. Kahn (1991), 'The Role of Demandable Debt in Structuring Optimal Banking Arrangements', American Economic Review 81, pp. 479-513

Chang, Roberto, and Andres Velasco (2000), 'Liquidity Crises in Emerging Markets: Theory and Policy', Federal Reserve Bank of Atlanta and NYU and NBER.

Chang, Roberto, and Andres Velasco (2001), 'A Model of Financial Crises in Emerging Markets', Quarterly Journal of Economics, Vol. 116 (2), pp. 489-517.

Chari, V V and Jagannathan, Ravi (1988), 'Banking Panics, Information, and Rational Expectations Equilibrium,' Journal of Finance, American Finance Association, Vol. 43(3) pp. 749-61.

Danielsson, Jon (2009), 'The first casualty of the crisis: Iceland, in The First Global Financial Crisis of the 21st Century', in The First Global Financial Crisis of the 21st Century, pp. 9-13. 
Diamond, Douglas W. and Raghuram G. Rajan, (2001), 'Liquidity Risk, Liquidity Creation, and Financial Fragility: A Theory of Banking,' Journal of Political Economy, Vol. 109(2), pp. 287-327.

Diamond, Douglas W and Raghuram G. Rajan (2000), 'A Theory of Bank Capital', Journal of Finance, Vol. LV(6), pp. 2431-2465.

Diamond, Douglas W. and Raghuram G. Rajan (2006), 'Money in a Theory of Banking', American Economic Review, American Economic Association, Vol. 96(1), pp. 30-53.

Diamond, Douglas, and Philip Dybvig (1983), 'Bank Runs, Deposit Insurance, and Liquidity,' Journal of Political Economy 91, pp 401-419.

Eichengreen, B., R. Hausmann and U. Panizza (2005), 'The Pain of Original Sin,' in B. Eichengreen and R. Hausmann (eds.) Other People's Money: Debt Denomination and Financial Instability in Emerging-Market Economies, University of Chicago Press.

Hardy, Daniel and Ceyla, Pazarbasioglu (1998), 'Leading Indicators of Banking Crises-Was Asia Different?', IMF Working Papers 98/91, International Monetary Fund.

Kaminsky, Graciela L. (1998), 'Currency and Banking Crises: The Early Warnings of Distress', FRB International Finance Discussion Paper No. 629.

Kaminsky, Graciela L. and Carmen M. Reinhart (1999), 'The Twin Crises: The Causes of Banking and Balance-Of-Payments Problems', American Economic Review, Vol. 89, No. 3, pp. 473-500.

Sirtaine, Sophie and Skamnelos, Ilias (2007), 'Credit growth in emerging Europe: a cause for stability concerns?', Policy Research Working Paper Series 4281, The World Bank.

Von Hagen, Jurgen and Siedschlag, Iulia (2008), 'Managing Capital Flows: Experiences from Central and Eastern Europe', Economic and Social Research Institute (ESRI), Papers WP234. 


\section{Documents de travail du BETA}

2010-01 The Aggregation of Individual Distributive Preferences through the Distributive Liberal Social Contract : Normative Analysis

Jean MERCIER-YTHIER, janvier 2010.

2010-02 Monnaie et Crise Bancaire dans une Petite Economie Ouverte Jin CHENG, janvier 2010.

La présente liste ne comprend que les Documents de Travail publiés à partir du $1^{\mathrm{er}}$ janvier 2010. La liste complète peut être donnée sur demande.

This list contains the Working Paper writen after January 2010, 1rst. The complet list is available upon request. 